\title{
Equação de Choquard: Existência de Solução de Energia Mínima
}

\author{
Colaboração Matemática Aberta*
}

10 de fevereiro de 2022

\begin{abstract}
Resumo
Neste trabalho, apresentamos um estudo sobre a existência de solução de energia mínima via método de Nehari para uma classe de problemas não locais envolvendo potenciais limitados ou ilimitados.
\end{abstract}

Palavras-chave: Equação de Choquard. Sequência de Cerami. Variedade de Nehari.

\section{Introdução}

1. O objetivo aqui é mostrar a existência de solução mínima via método de Nehari.

2. Note a seguinte equação de Choquard não linear

$$
\left\{\begin{array}{l}
-\triangle u+V(x) u=\left(\int_{\mathbb{R}^{N}} \frac{Q(y) F(u(y))}{|x-y|^{\mu}} d y\right) Q(x) f(u(x)) \\
u \in \mathcal{D}^{1,2}\left(\mathbb{R}^{N}\right),
\end{array}\right.
$$

onde $N \geq 3,0<\mu<N, V \in \mathcal{C}\left(\mathbb{R}^{N},[0,+\infty)\right), Q \in \mathcal{C}\left(\mathbb{R}^{N},(0,+\infty)\right)$, $f \in \mathcal{C}(\mathbb{R}, \mathbb{R})$ e $F(t)=\int_{0}^{t} f(s) d s$.

*Todos os autores com suas afiliações aparecem no final deste artigo. 
3. O funcional energia para o problema (2) é definido por

$$
\begin{aligned}
\Phi(u) & =\frac{1}{2} \int_{\mathbb{R}^{N}}\left[|\nabla u|^{2}+V(x) u^{2}\right] d x \\
& -\frac{1}{2} \int_{\mathbb{R}^{N}} \int_{\mathbb{R}^{N}} \frac{Q(x) Q(y) F(u(x)) F(u(y))}{|x-y|^{\mu}} d x d y .
\end{aligned}
$$

4. Propriedades sobre os potenciais $V$ e $Q$ :

(Q1) $V(x), Q(x)>0, V \in \mathcal{C}\left(\mathbb{R}^{N}, \mathbb{R}\right)$ e $Q \in \mathcal{C}\left(\mathbb{R}^{N}, \mathbb{R}\right) \cap L^{\infty}\left(\mathbb{R}^{N}, \mathbb{R}\right)$.

(Q2) Se $\left\{A_{n}\right\} \subset \mathbb{R}^{N}$ é uma sequência do conjunto de Borel, tal que a medida de Lebesgue para $A_{n}$ é menor do que $\delta, \forall n$ e algum $\delta>0$, então

$$
\lim _{r \rightarrow+\infty} \int_{A_{n} \cap B_{r}^{c}(0)}[Q(x)]^{\frac{2 N}{2 N-\mu}} d x=0 \text {, uniformemente em } n \in \mathbb{N} .
$$

(Q3) $\frac{Q}{V} \in L^{\infty}\left(\mathbb{R}^{N}\right)$.

(Q4) Existe $p \in\left(2,2^{*}\right)$ tal que

$$
\frac{[Q(x)]^{\frac{2 N}{2 N-\mu}}}{[V(x)]^{\frac{2^{*}-p}{2^{*}-2}}} \longrightarrow 0, \quad|x| \rightarrow+\infty .
$$

5. Vamos assumir as seguintes hipóteses sobre a não linearidade da função $f$ :

(F0) Se $f \in \mathcal{C}(\mathbb{R}, \mathbb{R})$,

$$
\lim _{t \rightarrow 0} \frac{F(t)}{|t|^{(2 N-\mu) / N}}=0 \quad \text { e } \lim _{|t| \rightarrow+\infty} \frac{F(t)}{|t|^{(2 N-\mu) /(N-2)}}=0 .
$$

Então, existe uma constante $C_{0}>0$ tal que

$$
|t f(t)| \leq C_{0}\left(|t|^{(2 N-\mu) / N}+|t|^{(2 N-\mu) /(N-2)}\right), \quad \forall t \in \mathbb{R} .
$$


(F1) $\lim _{|t| \rightarrow+\infty} \frac{F(t)}{|t|}=+\infty$

(F2) $\lim _{t \rightarrow 0} \frac{F(t)}{|t|^{\frac{2 N-\mu}{N}}}=0$, se está em (Q3); ou $\lim _{t \rightarrow 0} \frac{F(t)}{|t|^{\frac{p(2 N-\mu)}{2 N}}}=0$, se está em (Q4).

(F3) $\lim _{|t| \rightarrow+\infty} \frac{F(t)}{|t|^{\frac{2 N-\mu}{N-2}}}=+\infty$, se (Q3) é válido; ou $\lim _{|t| \rightarrow+\infty} \frac{F(t)}{|t|^{\frac{p(2 N-\mu)}{2 N}}}<+\infty$, se (Q4) é válido.

(F4) $f(t)$ é não-decrescente em $\mathbb{R}$.

6. Ressaltamos que $(2 N-\mu) /(N-2)$ e $(2 N-\mu) / N$ são, respectivamente, expoentes críticos superior e inferior.

\section{Regularidade do Funcional $\Phi$}

7. Desigualdade de Hardy-Littlewood-Sobolev: Sejam $t, r>1 \mathrm{e}$ $0<\mu<N \operatorname{com} 1 / t+1 / r+\mu / N=2, g \in L^{t}\left(\mathbb{R}^{N}\right), h \in L^{r}\left(\mathbb{R}^{N}\right)$. Então existe uma constante $C(t, N, \mu, r)$ independente de $g$ e $h$, tal que

$$
\int_{\mathbb{R}^{N}} \int_{\mathbb{R}^{N}} \frac{g(x) h(y)}{|x-y|^{\mu}} d x d y \leq C(t, N, \mu, r)\|g\|_{t}\|h\|_{r} .
$$

8. A desigualdade de Hardy-Littlewood-Sobolev, a Estimativa do Potencial de Riesz e o Teorema da Convergência Dominada de Lebesgue, essencialmente, nos ajudam a mostrar que $\Phi$ está bem definido e de $\mathcal{C}^{1}\left(H^{1}\left(\mathbb{R}^{N}\right) ; \mathbb{R}\right)$.

\section{Solução de Energia Mínima Via Método de Nehari}

9. Defina o conjunto de Nehari por

$$
\mathcal{N}:=\left\{u \in E \backslash\{0\}:\left\langle\Phi^{\prime}(u), u\right\rangle=0\right\} \neq \varnothing .
$$




\section{Lema 1}

10. Suponha que $(V, Q) \in \mathcal{K}$ e $f$ satisfaz (F2) e (F3).

11. Então

$$
\left|\int_{\mathbb{R}^{N}} \int_{\mathbb{R}^{N}} \frac{Q(x) Q(y) F(u(x)) F(u(y))}{|x-y|^{\mu}} d x d y\right|<+\infty, \quad \forall u \in E,
$$

e para $u \in E$, existe $\mathcal{C}_{1}>0$ tal que

$$
\left|\int_{\mathbb{R}^{N}} \int_{\mathbb{R}^{N}} \frac{Q(x) Q(y) F(u(x)) f(u(y)) v(y)}{|x-y|^{\mu}} d x d y\right|<\mathcal{C}_{1}\|v\|, \quad \forall v \in E .
$$

12. Além disso, se $\left\{u_{n}\right\} \subset E$ for uma sequência tal que $u_{n} \rightarrow u$ em $E$, então

$$
\lim _{n \rightarrow \infty} \int_{\mathbb{R}^{N}} \int_{\mathbb{R}^{N}} \frac{Q(x) Q(y)\left[F\left(u_{n}(x)\right) F\left(u_{n}(y)\right)-F(u(x)) F(u(y))\right]}{|x-y|^{\mu}}=0
$$

$\mathrm{e}$

$$
\lim _{n \rightarrow \infty} \int_{\mathbb{R}^{N}} \int_{\mathbb{R}^{N}} \frac{Q(x) Q(y) F\left(u_{n}(x)\right) f\left(u_{n}(y)\right)\left[u_{n}(y)-u(y)\right]}{|x-y|^{\mu}}=0 .
$$

13. Propriedades Adicionais:

$$
\begin{aligned}
& f(\tau) \geq 0, \text { se } \tau \geq 0 ; f(\tau) \leq 0, \text { se } \tau \leq 0 ; \\
& f(\tau) \tau \geq \int_{0}^{\tau} f(t) d t=F(\tau) \geq 0, \quad \forall \tau \in \mathbb{R} ; \\
& F(t) \geq 0, \forall t \geq 0 .
\end{aligned}
$$


14. Além disso, $\frac{F(\tau)}{\tau}$ é uma função monótona não-decrescente em $(-\infty, 0) \cup(0,+\infty)$.

15. A partir de agora, iremos enunciar alguns resultados que usamos no decorrer do trabalho. Para o leitor interessado nas demonstrações, referimos [2].

16. Suponha que (F2) e (F4) são válidos.

17. Então, para todo $t \geq 0$ e $\tau_{1}, \tau_{2} \in \mathbb{R}$;

$$
\begin{aligned}
& g\left(t, \tau_{1}, \tau_{2}\right):=F\left(t \tau_{1}\right) F\left(t \tau_{2}\right)-F\left(\tau_{1}\right) F\left(\tau_{2}\right) \\
& +\frac{1-t^{2}}{2}\left[F\left(\tau_{1}\right) f\left(\tau_{2}\right) \tau_{2}+F\left(\tau_{2}\right) f\left(\tau_{1}\right) \tau_{1}\right] \geq 0 .
\end{aligned}
$$

18. Suponha que $(V, Q) \in \mathcal{K}$ e $f$ satisfaz $(\mathrm{F} 2)-(\mathrm{F} 4)$.

19. Então,

$$
\Phi(u) \geq \Phi(t u)+\frac{1-t^{2}}{2}\left\langle\Phi^{\prime}(u), u\right\rangle ; \quad \forall u \in E, t \geq 0 .
$$

20. Lembre-se que

$$
\begin{aligned}
\left\langle\Phi^{\prime}(u), v\right\rangle & =\int_{\mathbb{R}^{N}}[\nabla u \cdot \nabla v+V(x) u v] d x \\
& -\int_{\mathbb{R}^{N}} \int_{\mathbb{R}^{N}} \frac{Q(x) Q(y) F(u(x)) f(u(y)) v(y)}{|x-y|^{\mu}} d x d y .
\end{aligned}
$$

21. Suponha que $(V, Q) \in \mathcal{K}$ e $f$ satisfaz $(\mathrm{F} 2)-(\mathrm{F} 4)$.

22. Então,

$$
\Phi(u)=\max _{t \geq 0} \Phi(t u), \quad \forall u \in \mathcal{N}
$$


23. Suponha que $(V, Q) \in \mathcal{K}$ e $f$ satisfaz $(\mathrm{F} 1)-(\mathrm{F} 4)$.

24. Então, para qualquer $u \in E \backslash\{0\}$, existe um único $t_{u}>0$, tal que $t_{u} u \in \mathcal{N}$.

25. Para compreender (24), observe o gráfico abaixo:

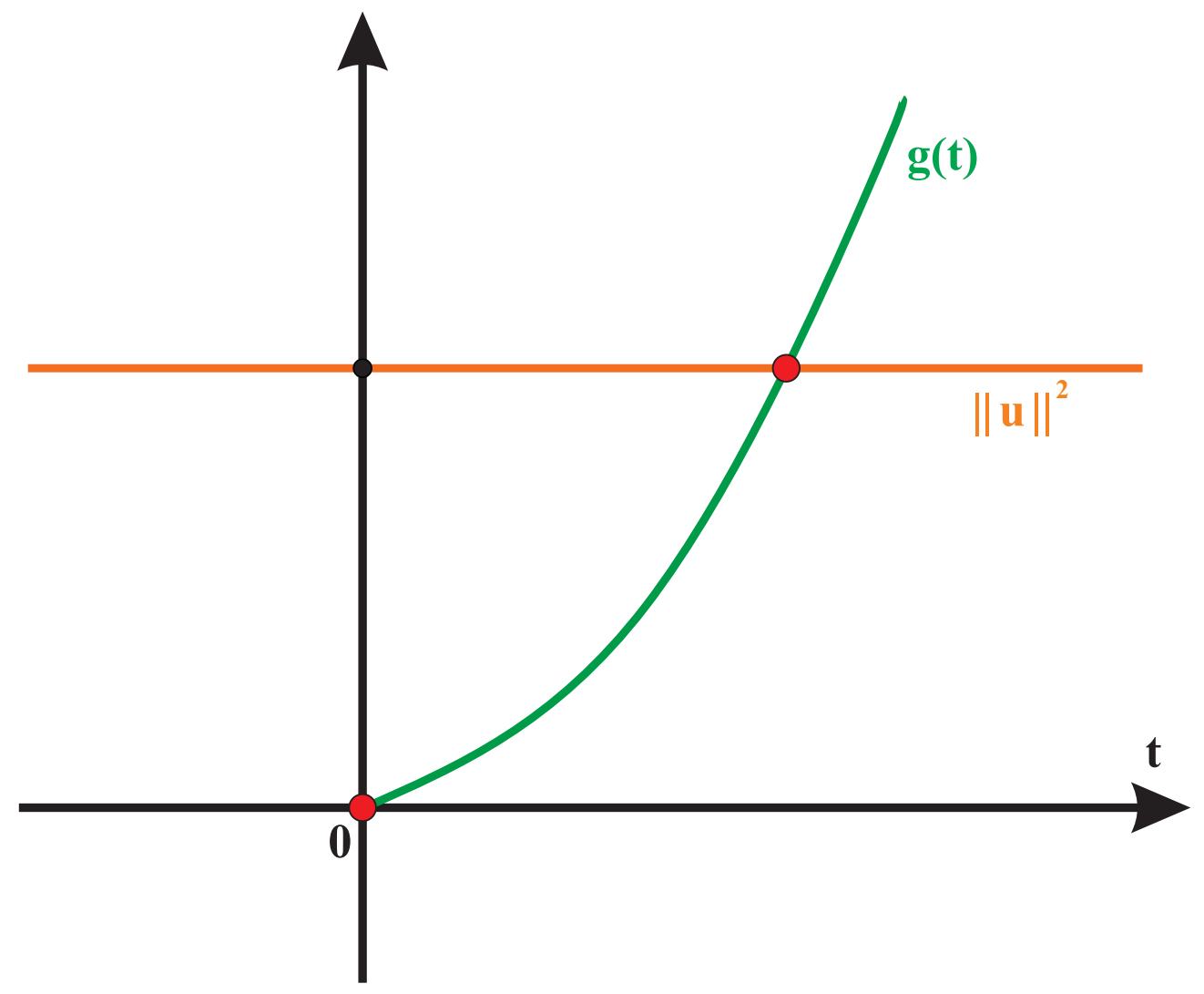

Figura 1: Existência de projeção única.

26. Suponha que $(V, Q) \in \mathcal{K}$ e $f$ satisfaz (F1)-(F4).

27. Então,

$$
\inf _{u \in \mathcal{N}} \Phi(u):=m=\inf _{u \in E \backslash\{0\}} \max _{t \geq 0} \Phi(t u)>0 .
$$

28. Sejam $X$ um espaço de Banach e $\Phi$ um funcional $\mathcal{C}^{1}(X, \mathbb{R})$.

29. Dizemos que $\left\{u_{n}\right\}$ é uma sequência de Cerami no nível $m$, quando

$$
\Phi\left(u_{n}\right) \rightarrow m, \quad\left(1+\left\|u_{n}\right\|\right)\left\|\Phi^{\prime}\left(u_{n}\right)\right\| \rightarrow 0 .
$$

30. Além disso, $\Phi$ satisfaz a condição de Cerami quando qualquer sequência de Cerami admite subsequência convergente. 
31. O nível $m$ é atingido por uma função não negativa, ou seja, existe $u \in \mathcal{N} ; u(x) \geq 0$ q.t.p em $\mathbb{R}^{N}$ de modo que

$$
\Phi(u)=m
$$

32. Suponha que $(V, Q) \in \mathcal{K}$ e $f$ satisfaz $(\mathrm{F} 1)-(\mathrm{F} 4)$.

33. Então existe uma constante $c_{*} \in(0, m]$ e uma sequência $\left\{u_{n}\right\} \subset E$ satisfazendo

$$
\Phi\left(u_{n}\right) \rightarrow c_{*}, \quad\left\|\Phi^{\prime}\left(u_{n}\right)\right\|\left(1+\left\|u_{n}\right\|\right) \rightarrow 0 .
$$

34. Demonstração: Seja $m=\inf _{u \in \mathcal{N}} \Phi(u)$.

35. Neste caso, podemos pegar uma sequência $\left\{v_{k}\right\} \in \mathcal{N}$ de modo que

$$
m \leq \Phi\left(v_{k}\right)<m+\frac{1}{k}, \quad k \in \mathbb{N} .
$$

36. Segue-se que $\Phi(u) \geq \delta_{0}>0, \forall u \in S_{\rho_{0}}=\left\{u \in E:\|u\|=\rho_{0}\right\}$.

37. A geometria do Passo da Montanha garante que existe $\left\{u_{k, n}\right\}_{n \in \mathbb{N}}$ tal que

$$
\Phi\left(u_{k, n}\right) \rightarrow c_{k}, \quad\left\|\Phi^{\prime}\left(u_{k, n}\right)\right\|\left(1+\left\|u_{k, n}\right\|\right) \rightarrow 0, \quad k \in \mathbb{N} \text { e } n \rightarrow+\infty .
$$

38. Note que $c_{k} \in\left[\delta_{0}, \sup _{t \geq 0} \Phi\left(t v_{k}\right)\right]$.

39. Logo, podemos escolher uma outra sequência $\left\{n_{k}\right\} \subset \mathbb{N}$ tal que

$$
\Phi\left(u_{k, n_{k}}\right) \in\left[\delta_{0}, m+\frac{1}{k}\right], \quad\left\|\Phi^{\prime}\left(u_{k, n_{k}}\right)\right\|\left(1+\left\|u_{k, n_{k}}\right\|\right)<\frac{1}{k}, k \in \mathbb{N} .
$$

40. Portanto, tomando $u_{k}=u_{k, n_{k}}, k \in \mathbb{N}$, obtemos

$$
\Phi\left(u_{k}\right) \rightarrow c_{*} \in\left[\delta_{0}, m\right], \quad\left\|\Phi^{\prime}\left(u_{k}\right)\right\|\left(1+\left\|u_{k}\right\|\right) \rightarrow 0, \quad k \rightarrow+\infty .
$$


41. Isso finaliza a demonstração.

42. Suponha que $(V, Q) \in \mathcal{K}$ e $f$ satisfaz $(\mathrm{F} 1)-(\mathrm{F} 4)$.

43. Então, a sequência $\left\{u_{n}\right\} \subset E$ satisfazendo

$$
\Phi\left(u_{n}\right) \rightarrow c \geq 0, \quad\left\langle\Phi^{\prime}\left(u_{n}\right), u_{n}\right\rangle \rightarrow 0
$$

é limitada em $E$.

\section{Teorema:}

44. Suponha que $(V, Q) \in \mathcal{K}$ e $f$ satisfaz $(\mathrm{F} 1)-(\mathrm{F} 4)$.

45. Então (2) tem uma solução de energia mínima $\bar{u} \in E$ tal que

$$
\Phi(\bar{u})=\inf _{\mathcal{N}} \Phi>0 .
$$

46. Demonstração: (31), (33) e (43) fornecem uma sequência limitada $\left\{u_{n}\right\} \subset E$.

47. Passando para uma subsequência, o Teorema de Banach-Alaoglu assegura que $u_{n} \rightarrow u \mathrm{em} E$.

48. Dessa forma, obtemos

$$
\left\|u_{n}-u\right\|^{2}=o_{n}(1)
$$

49. A equação (48) garante que $u_{n} \rightarrow u$ em $H^{1}\left(\mathbb{R}^{N}\right)$.

50. Além disso,

$$
\left\|\Phi^{\prime}\left(u_{n}\right)\right\| \leq \frac{\varepsilon}{1+\left\|u_{n}\right\|} \leq \varepsilon, \quad \forall n \geq n_{0} .
$$

51. Pela arbitrariedade de $\varepsilon>0$, temos que

$$
\Phi^{\prime}(u)=0 \Leftrightarrow \Phi^{\prime}(u) h=0, \quad \forall h \in H^{1}\left(\mathbb{R}^{N}\right) .
$$


52. $\log 0, \Phi(u)=c_{*} \in(0, m]$.

53. Assim, dado $u \in \mathcal{N}$, segue que $\Phi(u) \geq m$ e $\Phi(u) \leq m$.

54. Isso implica que

$$
\Phi(u)=m>0, \quad \forall u \in E .
$$

55. Portanto, $u \in E$ é uma solução de energia mínima para (2).

\section{Considerações Finais}

56. Assim fica provado o teorema desejado.

\section{Ciência Aberta}

O arquivo tex para este artigo, juntamente com outros arquivos suplementares, estão disponíveis em [4].

\section{Referências}

[1] CHEN, S.; YUAN, S. Ground state solutions for a class of Choquard equations with potential vanishing at infinity, J. Math. Appl. 463: 880-894, 2018.

[2] Lima, E. D. Equação de Choquard: existência de soluções de energia mínima para uma classe de problemas não locais envolvendo potenciais limitados ou ilimitados, dissertação de mestrado, UFG, Goiânia, 2020 .

[3] BADIALE, M.; SERRA, E. Semilinear Elliptic Equations for Begginers. British Library Cataloguing in Publication Data, 2011.

[4] Lobo, Matheus P. "Simple Guidelines for Authors: Open Journal of Mathematics and Physics." OSF Preprints, 15 Nov. 2019. https://doi.org/10.31219/osf.io/fk836. 


\section{Colaboração Matemática Aberta}

Eduardo Dias Lima

(autor principal,duardo.dias16@hotmail.com) 1,2 